\title{
CARNITINE INSUFFICIENCY IS ASSOCIATED WITH ADVERSE OUTCOMES IN PATIENTS WITH HEART FAILURE WITH PRESERVED EJECTION FRACTION
}

\author{
Y. Kinugasa, S. Sugihara, K. Yamada, M. Miyagi, K. Matsubara, M. Kato, K. Yamamoto
}

\begin{abstract}
Background: L-carnitine is an essential nutrient that plays a vital role in fatty acid energy metabolism of the heart and skeletal muscles. Primary or secondary carnitine insufficiency contributes to progressive left ventricular systolic dysfunction and physical frailty. However, the clinical features of patients with heart failure with preserved ejection fraction (HFpEF) and carnitine insufficiency remain unclear. Objectives: The present study aimed to evaluate the clinical characteristics and outcomes of these patients. Design: A prospective cohort study. Setting: Tottori University hospital. Participants: 117 patients who were hospitalized with HFpEF (ejection fraction $\geq 45 \%$ ). Measurement: All measurements were obtained at hospital discharge. Carnitine insufficiency was defined as the lowest quantile of free carnitine level $(<56.3 \mu \mathrm{mol} / \mathrm{L})$ or the highest quantile of acylcarnitine to free carnitine ratio ( $\geq 0.35$ ). Nutritional status and physical activity were assessed by the Geriatric Nutritional Risk Index (GNRI) and Barthel index (BI). Left ventricular diastolic function was assessed by echocardiography. The composite endpoints were hospitalization for heart failure and death from cardiac causes. Results: Patients with carnitine insufficiency (44.4\%) had lower values of GNRI and BI, higher B-type natriuretic peptide levels, and lower early diastolic mitral annular velocity in the subgroups with sinus rhythm compared with those with preserved carnitine (all $\mathrm{p}<0.05$ ). During a mean follow-up of $472 \pm 249$ days, composite endpoints occurred in $26.5 \%$ of patients. Multivariate Cox hazard analysis showed that carnitine insufficiency was an independent predictor of cardiac events $(\mathrm{p}<0.05)$. Conclusions: Carnitine insufficiency is associated with adverse outcomes in patients with HFpEF.
\end{abstract}

Key words: L-carnitine, $\mathrm{HFpEF}$, frailty, left ventricular diastolic function, malnutrition.

\section{Introduction}

Malnutrition is common in patients with heart failure with preserved ejection fraction (HFpEF) (1). Poor nutrition leads to the development of sarcopenia and frailty, which can cause physical functional decline, decreased quality of life, and poor prognosis (2). Despite the clinical significance of nutritional problems, important nutritional factors to target intervention remain undefined.

L-carnitine is an essential nutrient that is present in red meat, and it plays a vital role in fatty acid energy metabolism of the heart and skeletal muscles (3). Several metabolic disorders, including carnitine transporter (organic cation transporter 2; OCTN2) mutation, poor diet or malabsorption of carnitine, increased renal tubular losses (Fanconi syndrome), haemodialysis, and certain

Division of Cardiovascular Medicine, Department of Molecular Medicine and Therapeutics, Faculty of Medicine, Tottori University, Yonago, Japan

Corresponding Author: Yoshiharu Kinugasa, M.D., Ph.D. Division of Cardiovascular Medicine, Department of Molecular Medicine and Therapeutics, Faculty of Medicine, Tottori University 36-1 Nishi-cho, Yonago 683-8504, Japan, Phone: +81 859-38-6517 Fax: +81-859-38-6519 E-mail: ykinugasa-circ@umin.ac.jp drugs, can cause carnitine insufficiency (3). Primary or secondary carnitine insufficiency contributes to the development of left ventricular systolic dysfunction and muscle weakness $(3,4)$. Several studies have also shown the beneficial effect of carnitine supplementation on patients with carnitine insufficiency $(3,4)$.

Our recent study showed decreased free carnitine levels in plasma and left ventricular tissue in a hypertensive HFpEF model rat using quantitative metabolome analysis (5). Carnitine insufficiency is likely to result from acquired downregulation of OCTN2. L-carnitine supplementation in an animal rat model prevented progression of left ventricular fibrosis and stiffening and development of $\mathrm{HF}$, resulting in an improvement of survival rate (5). However, clinical features of human HFpEF with carnitine insufficiency remains unclear. The present study aimed to evaluate the clinical characteristics and outcomes of patients with HFpEF and L-carnitine insufficiency. 
CARNITINE INSUFFICIENCY IS ASSOCIATED WITH ADVERSE OUTCOMES IN PATIENTS WITH HEART FAILURE WITH PRESERVED EJECTION FRACTION

\section{Methods}

\section{Patients}

The present study consecutively enrolled 152 patients who were hospitalized and discharged from Tottori University Hospital with the primary diagnosis of HFpEF from January 2012 to May 2015. Among them, 35 patients were excluded because their informed consent was not obtained. Finally, a total of 117 patients were enrolled in this study.

Patients with HFpEF were defined as follows: 1) patients with symptoms and signs of HF as defined by the Framingham criteria, 2) patients with a left ventricular ejection fraction $\geq 45 \%$ as previously described $(6,7)$, and 3) patients without HF aetiologies of severe valve disease, congenital disease, complete atrial ventricular block, sick sinus syndrome, pericardial disease, primary pulmonary hypertension, pulmonary artery embolism, and acute myocardial infarction. Patients under chronic dialysis were also excluded.

\section{Data collection}

Medical records were reviewed for demography, medical history, comorbidities, laboratory data, echocardiograms, medications, and clinical course. All measurements were taken at discharge. Echocardiographic data were measured by one experienced sonographer as recommended by the American Society of Echocardiography (8). Early diastolic mitral annular velocity $\left(E^{\prime}\right)$ was obtained from the septal site of the mitral leaflet. Early diastolic mitral inflow (E) and $E^{\prime}$ in atrial fibrillation (Af) was measured from the cardiac cycle with regular beats. $\mathrm{E}^{\prime}$ was available in 112 patients. The estimated glomerular filtration rate was calculated as previously described (9). Follow-up data were obtained from medical records or a telephone interview. We evaluated the composite endpoints of cardiac-cause death and unplanned re-hospitalization for HF during the follow-up period.

\section{Measurement of serum L-carnitine levels}

Serum L-carnitine levels were measured with enzymatic cycling methods (carnitine assay kit; Kainos Laboratories Co., Tokyo, Japan) as previously described (5). L-carnitine is present mainly in the form of free carnitine (FC) and acylcarnitine (AC). Carnitine insufficiency was defined as a reduced FC level or increased $\mathrm{AC}$ to $\mathrm{FC}$ ratio, which indicates the presence of abnormal carnitine metabolism, reflecting relative carnitine deficiency to increased demand of fatty acid metabolism as previously described (10). Because normal carnitine levels have not been established in patients with $\mathrm{HF}$, the present study defined carnitine insufficiency as the lowest quantile of FC level $(<56.3 \mu \mathrm{mol} / \mathrm{L})$ or the highest quantile of $\mathrm{AC}$ to $\mathrm{FC}$ ratio $(\geq 0.35)$ in this cohort.

\section{Geriatric Nutritional Risk Index}

The Geriatric Nutritional Risk Index (GNRI) was recently postulated as a nutritional risk screening tool in patients with HFpEF (1. 11). The GNRI was calculated from serum albumin levels and body mass index (BMI) as previously described (1).

\section{Activities of daily living}

The Barthel index (BI) was measured by nurses and physical therapists at discharge from hospital. The BI measures a patient's functional status for basic daily activities, with scores ranging from 0 (total dependence) to 100 points (independent patients) $(12,13)$.

\section{Clinical outcomes}

We evaluated mortality from cardiac causes and unplanned re-hospitalization for HF during the follow-up period. Death from a cardiac cause was defined as death due to HF and sudden death.

The investigation conforms to the principles outlined in the Declaration of Helsinki. The study was approved by the research ethical committee of Tottori University. Written informed consent was provided by each subject.

\section{Statistical analysis}

Continuous variables are expressed as means \pm standard deviation for normally distributed variables, and median and interquartile ranges for non-normally distributed variables. The normality of distribution was assessed by the Kolmogorov-Smirnov test. Differences in continuous variables were compared using the t-test for normally distributed variables and the Mann-Whitney U test for non-normally distributed variables. Categorical variables were compared using the $\chi 2$ test. The event-free survival curve after hospital discharge was estimated by the Kaplan-Meier method and compared using the log-rank test. Cox proportional hazards models were used to assess the effect of reduced L-carnitine levels on the primary outcome and its interaction with each subgroup. Independent predictors of cardiac events were assessed by stepwise regression with forward selection. All baseline variables that were associated with the composite endpoints in univariate analysis $(p<0.05)$ were entered into the model. A $p$ value $<0.05$ was considered statistically significant. All analyses were performed using IBM SPSS Statistics version 20 (SPSS, Inc., Chicago, IL, USA) and EZR (Saitama Medical Center, Jichi Medical University), which is a graphical user interface for $\mathrm{R}$ (The $\mathrm{R}$ Foundation for Statistical Computing, version 2.13.0) (1). 
Table 1

Baseline Characteristics of Patients with or without Carnitine Insufficiency

\begin{tabular}{|c|c|c|c|c|}
\hline & Overall $(n=117)$ & $\begin{array}{c}\text { Preserved } \\
\text { carnitine }(n=65)\end{array}$ & $\begin{array}{c}\text { Carnitine } \\
\text { insufficiency }(n=52)\end{array}$ & p value \\
\hline Age (years) & $79(72-83)$ & $79(71-82)$ & $80(72-83)$ & 0.359 \\
\hline Male (\%) & 52.1 & 55.4 & 48.1 & 0.549 \\
\hline Systolic blood pressure (mmHg) & $120 \pm 18$ & $119 \pm 15$ & $121 \pm 21$ & 0.626 \\
\hline Heart rate (beats/min) & $65 \pm 12$ & $65 \pm 11$ & $66 \pm 14$ & 0.726 \\
\hline Prior heart failure hospitalization $(\%)$ & 29.1 & 26.2 & 32.7 & 0.569 \\
\hline \multicolumn{5}{|l|}{ Nutritional status } \\
\hline $\mathrm{BMI}\left(\mathrm{kg} / \mathrm{m}^{2}\right)$ & $21.5 \pm 3.9$ & $21.9 \pm 4.0$ & $21.1 \pm 3.7$ & 0.240 \\
\hline Albumin (g/dl) & $3.6 \pm 0.4$ & $3.6 \pm 0.5$ & $3.5 \pm 0.4$ & 0.095 \\
\hline GNRI & $91.5 \pm 8.0$ & $92.9 \pm 8.0$ & $89.7 \pm 7.6$ & 0.032 \\
\hline \multicolumn{5}{|l|}{ Functional status } \\
\hline NYHA class III / IV (\%) & 12.0 & 9.2 & 15.4 & 0.464 \\
\hline Barthel index & $90(75-100)$ & $95(85-100)$ & $85(69-100)$ & 0.005 \\
\hline \multicolumn{5}{|l|}{ Comorbidity condition } \\
\hline Coronary artery disease $(\%)$ & 35.9 & 29.2 & 44.2 & 0.137 \\
\hline Hypertension (\%) & 82.1 & 78.5 & 86.5 & 0.374 \\
\hline Atrial fibrillation (\%) & 37.6 & 33.8 & 42.3 & 0.455 \\
\hline Diabetes (\%) & 43.6 & 46.2 & 40.4 & 0.662 \\
\hline Dyslipidemia (\%) & 41.9 & 41.5 & 42.3 & 1.000 \\
\hline COPD $(\%)$ & 21.4 & 23.1 & 19.2 & 0.782 \\
\hline Cerebrovascular disease $(\%)$ & 13.7 & 13.8 & 13.5 & 1.000 \\
\hline \multicolumn{5}{|l|}{ Laboratory values } \\
\hline Hemoglobin (g/dl) & $11.4 \pm 2.1$ & $11.7 \pm 2.2$ & $11.1 \pm 1.8$ & 0.123 \\
\hline Sodium (mEq/) & $139 \pm 3$ & $139 \pm 3$ & $140 \pm 4$ & 0.191 \\
\hline BUN (mg/dl) & $27.4(21.3-42.0)$ & $27.3(22.0-43.4)$ & $28.0(21.1-39.3)$ & 0.874 \\
\hline eGFR (ml/ml/1.73m2) & $45.1 \pm 21.6$ & $47.9 \pm 21.2$ & $41.7 \pm 21.9$ & 0.124 \\
\hline $\mathrm{BNP}(\mathrm{pg} / \mathrm{ml})$ & $168(76-348)$ & $130(68-288)$ & $244(140-376)$ & 0.013 \\
\hline $\mathrm{FC}(\mu \mathrm{mol} / \mathrm{L})$ & $69.4 \pm 20.5$ & $78.8 \pm 18.8$ & $57.6 \pm 16.2$ & $<0.001$ \\
\hline $\mathrm{AC}(\mu \mathrm{mol} / \mathrm{L})$ & $18.9 \pm 9.2$ & $17.0 \pm 5.9$ & $21.3 \pm 11.8$ & 0.012 \\
\hline $\mathrm{AC}$ to $\mathrm{FC}$ ratio & $0.25(0.20-0.35)$ & $0.21(0.18-0.25)$ & $0.36(0.26-0.40)$ & $<0.001$ \\
\hline \multicolumn{5}{|l|}{ Medication } \\
\hline ACE-I/ ARB (\%) & 88.0 & 89.2 & 86.5 & 0.874 \\
\hline$\beta$ blocker $(\%)$ & 80.3 & 80.0 & 80.8 & 1.000 \\
\hline Mineralocorticoid blocker (\%) & 35.9 & 32.3 & 40.4 & 0.477 \\
\hline Loop diuretics $(\%)$ & 92.3 & 87.7 & 98.1 & 0.081 \\
\hline
\end{tabular}

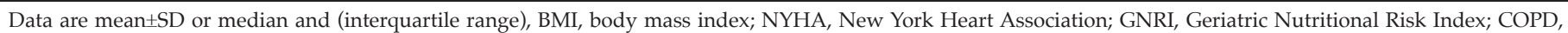

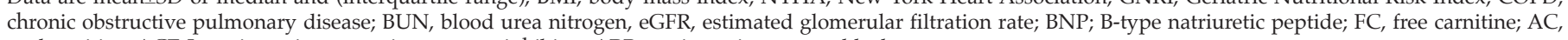
acylcarnitine; ACE-I, angiotensin-converting enzyme inhibitor, ARB, angiotensin receptor blocker. 


\section{Results}

\section{Baseline patients' characteristics}

Baseline characteristics of the patients are shown in Table 1 . The mean age of the overall cohort was $76 \pm 12$ years and $52.1 \%$ were male. The quantile values of FC levels were $<56.3,56.3-66.9,67.0-78.2$, and $>78.2$, and those of the AC to FC ratio were $<0.20,0.20-0.25,0.26$ 0.35 , and $>0.35$, respectively.

\section{Figure 1}

The Effect of Carnitine Insufficiency on Barthel Index and Early Diastolic Mitral Annular Velocity

A: Increased Prevalence of Carnitine Insufficiency with a Decrease in the Barthel Index.

B: The Relationship between Carnitine Insufficiency and Early Diastolic Mitral Annular Velocity in Patients with Sinus Rhythm

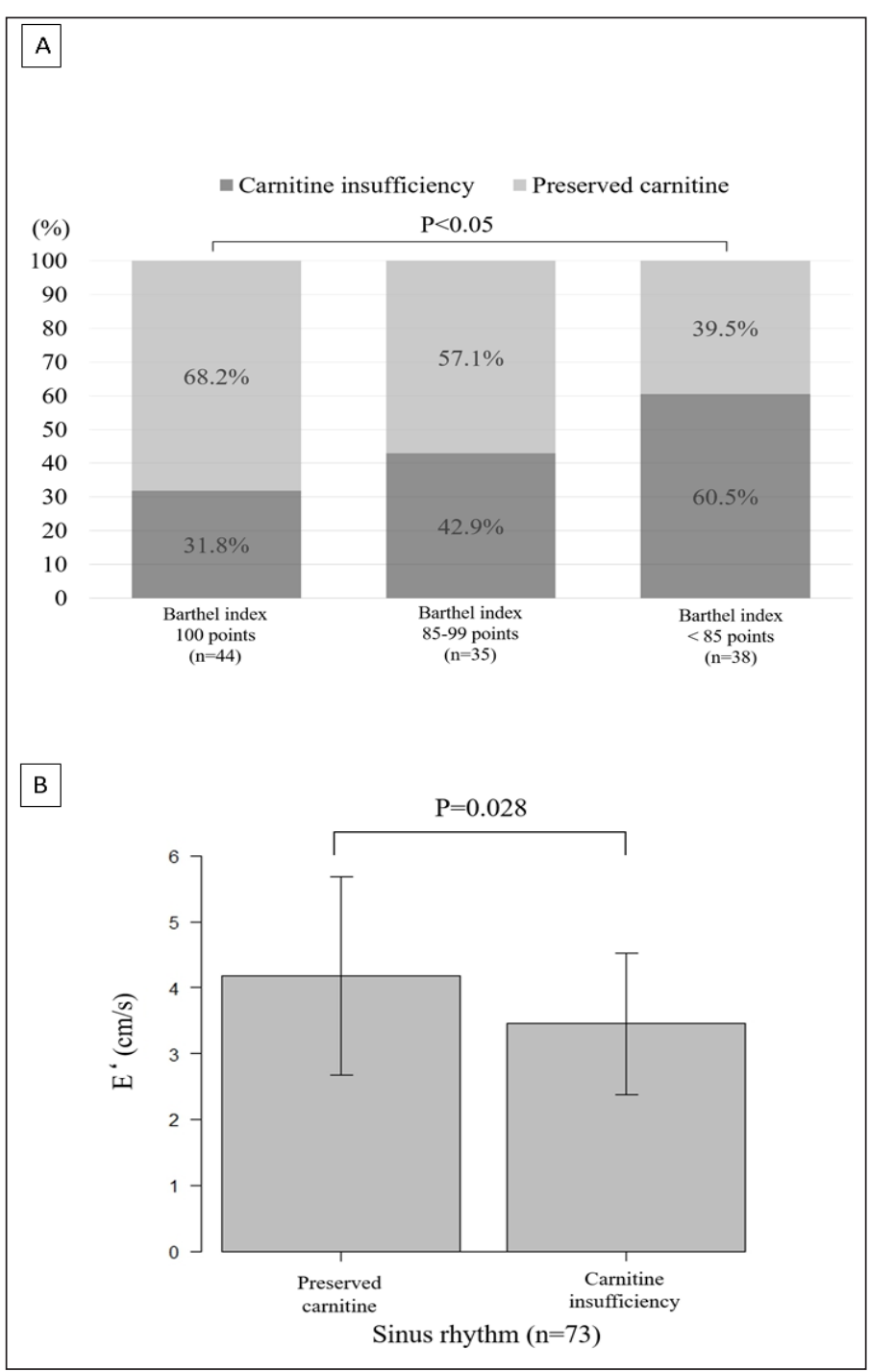

Figure 2

The Effect of Carnitine Insufficiency on Clinical Outcomes A: Event-free Survival Rate in Patients with or without Carnitine Insufficiency.

B: Effect of Carnitine Insufficiency on Primary Outcomes in the Subgroups

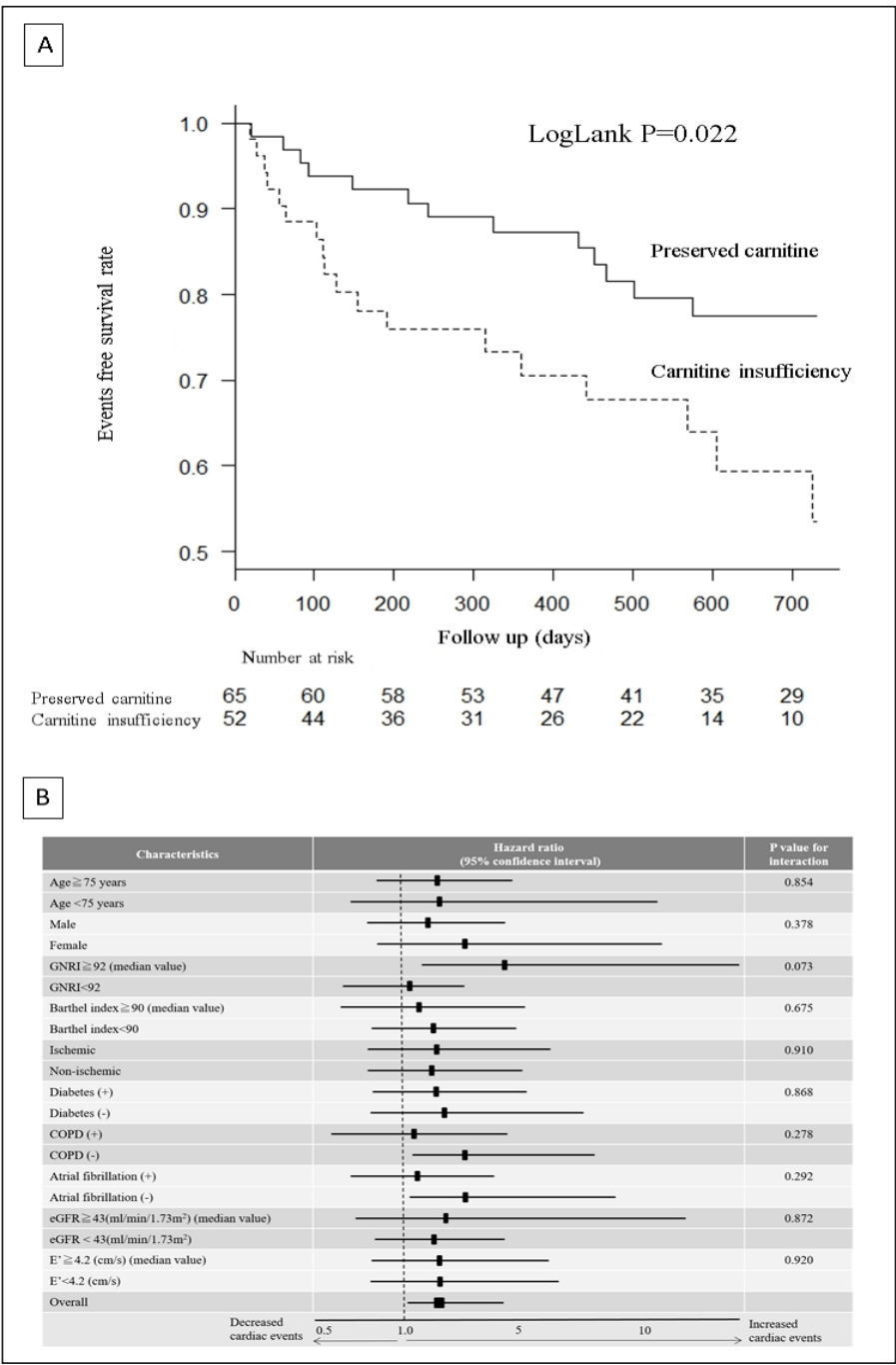

Patients with carnitine insufficiency, which was defined as the lowest quantile of FC level $(<56.3 \mu \mathrm{mol} / \mathrm{L})$ or the highest quantile of the AC to FC ratio $(\geq 0.35)$, had poor nutritional status as assessed by the GNRI and lower functional status as assessed by the BI than those with preserved carnitine (Table 1). Figure 1-A shows the prevalence of reduced L-carnitine levels according to the tertile values of the BI. Patients with the lowest tertile value of $\mathrm{BI}(\mathrm{BI}<85)$ had a two-fold increased prevalence of carnitine insufficiency compared with those with the highest BI $(B I=100)$. Patients with carnitine insufficiency had also significantly higher B-type natriuretic peptide levels $(p<0.05)$ and a marginally higher use of loop diuretics compared with those with preserved carnitine. Table 2 shows echocardiographic parameters between the two groups. $E^{\prime}$ tended to be lower in patients with carnitine insufficiency than in those with preserved carnitine $(p=0.05)$. The presence of Af was likely to affect 
Table 2

Echocardiographic Parameters in Patients with or without Carnitine Insufficiency

\begin{tabular}{|c|c|c|c|c|}
\hline & $\begin{array}{l}\text { Overall } \\
(n=117)\end{array}$ & $\begin{array}{c}\text { Preserved } \\
\text { carnitine } \\
(n=65)\end{array}$ & $\begin{array}{c}\text { Carnitine } \\
\text { insufficiency } \\
(n=52)\end{array}$ & $p$ value \\
\hline LVDd (mm) & $47.5 \pm 7.1$ & $47.2 \pm 5.9$ & $48.0 \pm 8.4$ & 0.510 \\
\hline LV mass index $\left(\mathrm{g} / \mathrm{m}^{2}\right)$ & $134.0 \pm 38.7$ & $131.9 \pm 41.1$ & $136.7 \pm 35.7$ & 0.502 \\
\hline RWT & $0.49 \pm 0.12$ & $0.49 \pm 0.12$ & $0.49 \pm 0.10$ & 0.854 \\
\hline $\operatorname{LVEF}(\%)$ & $58.0 \pm 8.7$ & $58.8 \pm 8.3$ & $56.9 \pm 9.0$ & 0.253 \\
\hline LAD & $46.4 \pm 8.8$ & $46.1 \pm 7.8$ & $46.8 \pm 9.9$ & 0.674 \\
\hline $\mathrm{E} / \mathrm{A}$ ratio & $0.77(0.61-1.07)$ & $0.78(0.65-1.14)$ & $0.76(0.53-0.97)$ & 0.195 \\
\hline DcT & $236(200-288)$ & $239(207-290)$ & $229(193-267)$ & 0.263 \\
\hline $\mathrm{E}^{\prime}$ & $4.2 \pm 1.5$ & $4.5 \pm 1.6$ & $3.9 \pm 1.4$ & 0.050 \\
\hline $\mathrm{E} / \mathrm{E}^{\prime}$ ratio & $18.5(13.6-23.7)$ & $17.5(13.3-22.5)$ & $19.4(13.8-27.3)$ & 0.232 \\
\hline TRPG (mmHg) & $24(19-29)$ & $23(19-29)$ & $25(19-29)$ & 0.421 \\
\hline IVC diameter (mm) & $16.0 \pm 3.9$ & $15.9 \pm 3.9$ & $16.1 \pm 3.8$ & 0.822 \\
\hline IVC respiratory change $(\%)$ & $49.9 \pm 13.0$ & $48.0 \pm 11.7$ & $52.2 \pm 14.1$ & 0.086 \\
\hline
\end{tabular}

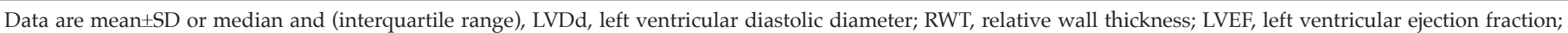
LAD, left atrial diameter; E, early diastolic mitral inflow; E', early diastolic annular velocity; TRPG, tricuspid regurgitation pressure gradient ; IVC, inferior vena cava.

Table 3

Cox Hazard Analysis for Independent Predictors of Cardiac Events

\begin{tabular}{lccc}
\hline & \multicolumn{2}{c}{ Univariate analysis } & \multicolumn{2}{c}{ Stepwise multivariate analysis } \\
& HR (95\% CI) & P value & HR (95\% CI) \\
\hline Carnitine insufficiency & $2.257(1.101-4.623)$ & 0.026 & $2.392(1.165-4.911)$ \\
Prior HF holue & $<.017$ \\
NYHAIII / IV & $3.459(1.704-7.022)$ & $<0.001$ & \\
Haemoglobin & $2.912(1.250-6.782)$ & 0.013 & \\
eGFR & $0.768(0.637-0.923)$ & 0.006 & $1.028(1.016-1.040)$ \\
BUN & $0.959(0.938-0.982)$ & $<0.001$ & $<0.001$ \\
BNP & $1.027(1.016-1.039)$ & $<0.001$ & 0.048 \\
\hline
\end{tabular}

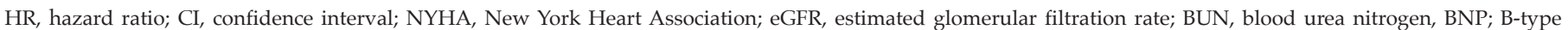
natriuretic peptide.

the assessment of left ventricular diastolic function owing to irregularity of the cycle length (14). Therefore, we excluded patients with Af from the analysis, and found that $E^{\prime}$ was significantly lower in patients with carnitine insufficiency than in those with preserved carnitine in the subgroup with sinus rhythm (Figure 1-B).

\section{Cardiac events}

During the mean follow-up of $472 \pm 249$ days, the composite endpoints of death due to cardiac causes and hospitalization for HF occurred in 31 (26.5\%) patients. The specific distribution of the composite endpoints was as follows: hospitalization for HF, 28 (23.9\%) patients; and death due to cardiac causes, three (2.6\%) patients. Kaplan-Meier event-free survival curves showed that patients with reduced carnitine insufficiency had a significantly higher frequency of cardiac events compared with those with preserved carnitine (Figure 2-A). In stepwise Cox hazard analysis, carnitine insufficiency was an independent predictor of the composite endpoints, as well as high blood urea nitrogen levels (Table 3). We also examined whether the effect of carnitine insufficiency on cardiac events was modified by age, sex, left ventricular diastolic function, nutritional status, physical function, and comorbidities (Figure 2-B). There was no significant interaction for the effect of carnitine insufficiency on the primary outcome. 


\section{Discussion}

The present study showed that carnitine insufficiency was associated with impaired left ventricular diastolic function, low physical activity, and increased future cardiac events in patients with HFpEF.

We found that poor nutritional status, as assessed by the GNRI, was significantly associated with carnitine insufficiency. Malnutrition is often accompanied by loss of muscle mass with sarcopenia and frailty (2). We and others have reported that a low GNRI is associated with surrogate markers of sarcopenia, including impaired muscle strength (6) and muscle mass (15). More than $90 \%$ of carnitine in the body is stored in cardiac and skeletal muscles (4). Therefore, decreased body carnitine stores accompanied by muscle loss may be responsible for carnitine insufficiency. Another potential reason for carnitine insufficiency is downregulation of OCTN2, which transports free carnitine into cells (3). L-carnitine levels decrease with advancing age because of an ageassociated reduction in OCTN2 expression $(16,17)$. OCTN2 downregulation has also been found in an HFpEF rat model (5). These findings may partially explain why HFpEF is a common HF phenotype in the elderly population.

L-carnitine plays an important role in fatty acid energy metabolism in the heart and skeletal muscles by transporting long-chain fatty acids into the mitochondria (3). Primary or secondary carnitine insufficiency is associated with muscle weakness (3). In the present study, we found that carnitine insufficiency was accompanied by low physical activity, as assessed by the Barthel index, in HFpEF patients. L-carnitine-associated abnormal energy metabolism in the skeletal muscle may lead to diminished muscle strength, attenuated endurance, and reduced physiologic function, which may increase vulnerability for developing functional dependency and/or death (16).

Impaired left ventricular diastolic function is a pathophysiology that determines prognosis in patients with HFpEF (18). We found that carnitine insufficiency was associated with impaired left ventricular diastolic function, which may have been another reason for the the relationship between L-carnitine insufficiency and poor outcomes. Our previous experimental study showed that L-carnitine prevents the development of left ventricular fibrosis and stiffening at least partly through enhanced production of prostacyclin in the hypertensive HFpEF rat model (5). Our experimental and clinical findings suggest that L-carnitine may play a major role in the development and progression of HFpEF.

Previous studies have reported some beneficial effects of L-carnitine supplementation in patients with HF. A previous randomized, double-blind, placebocontrolled trial evaluated the beneficial effect of L-carnitine supplementation on exercise capacity (19). Overall, exercise tolerance was not different between treated and non-treated groups. However, in patients with less reduced left ventricular ejection fraction values $(>30 \%)$, exercise tolerance was significantly improved in the treated group. Furthermore, a recent study with 29 HFpEF patients showed that L-carnitine supplementation for 3 months improved some measures of diastolic function compared with baseline (20). These results suggest that L-carnitine supplementation is a potential therapeutic strategy in patients with HFpEF. However, a recent study showed that intestinal microbiota metabolism of L-carnitine, trimethylamine-N-oxide (TMAO), promotes atherosclerosis and increases the risk of cardiovascular disease (21). In addition, elevated serum TMAO levels are associated with severity of disease and adverse outcome in patients with HF (22). Further investigations are necessary to evaluate the safety and efficiency of L-carnitine supplementation in patients with HFpEF.

Our study has several limitations. There is no obvious definition of carnitine insufficiency in patients with HF, but mean FC levels in healthy subjects was reported to be $49.4 \pm 13.0 \mu \mathrm{mol} / \mathrm{L}(23)$, indicating that the values below the mean level might be candidate value for carnitine insufficiency. However, FC is excreted from kidney, and its serum levels was affected by renal function (4). Patients with HFpEF often had chronic kidney disease, and renal impairment raises FC levels. Thus, the application of normal FC levels in healthy subjects may lead to underestimation of carnitine insufficiency, and specific criteria is likely to be needed for patients with HFpEF. Another candidate definition of carnitine insufficiency is AC to $\mathrm{FC}$ ratio $>0.4$ as previously described (4). However, its criteria focused on patients with dialysis who often had relatively high AC to FC ratio (4), and its application may also lead to underestimation of carnitine insufficiency. Therefore, in the present study, the definition of carnitine insufficiency was applied by the quantile value of $\mathrm{FC}$ and $\mathrm{AC}$ to $\mathrm{FC}$ ratio that were reliable abnormal value in the study population. However, our study population is relatively small, and further investigations are necessary to determine the definition of carnitine insufficiency in HFpEF. Second limitation was that we had no data on L-carnitine levels in skeletal muscle. Several studies have shown carnitine deficiency in skeletal muscle, regardless of normal plasma levels (4). However, assessment of the $\mathrm{AC}$ to $\mathrm{FC}$ ratio may attenuate the effects of a lack of data of tissue L-carnitine levels. There were also no data on TMAO and its association with L-carnitine levels and clinical outcomes. Furthermore, we could not assess the relationship between carnitine insufficiency and muscle mass, muscle strength, and exercise performance. Finally, this study was a single-center study with a relatively small sample size. Further investigations are necessary to confirm the effect of carnitine insufficiency on physical performance and clinical outcomes in another large cohort.

Carnitine insufficiency is associated with adverse 


\section{outcomes in patients with HFpEF.}

Acknowledgement: We thank for HF-team in Tottor university hospital.

Conflict of interest: Y Kinugasa received the grant from the Japan Society for the Promotion of Science (JSPS KAKENHI Grant no. 60598944) during the conduct of the study. K Yamamoto received the grants from the Japan Society for the Promotion of Science (JSPS KAKENHI Grant no. 25461058) during the conduct of the study, and grants and personal fees from Otsuka Phamaceutical Co. Ltd outside the submitted work.

Ethical standard: The investigation conforms to the princiles outlined in the declaration of Helsinki. The study was approved by the research ethnical committee of Tottori University.

\section{References}

1. Kinugasa Y, Kato M, Sugihara S, Hirai M, Yamada K, Yanagihara K, Yamamoto K. Geriatric nutritional risk index predicts functional dependency and mortality in patients with heart failure with preserved ejection fraction. Circ J. 2013;77(3):705-711.

2. Fried LP, Tangen CM, Walston J, Newman AB, Hirsch C, Gottdiener J, Seeman T, Tracy R, Kop WJ, Burke G, McBurnie MA, Cardiovascular Health Study Collaborative Research G. Frailty in older adults: evidence for a phenotype. J Gerontol A Biol Sci Med Sci. 2001 Mar;56(3):M146-156.

3. Flanagan JL, Simmons PA, Vehige J, Willcox MD, Garrett Q. Role of carnitine in disease. Nutr Metab (Lond). 2010;7:30.

4. Ahmad S. L-carnitine in dialysis patients. Semin Dial. 2001 MayJun;14(3):209-217.

5. Omori Y, Ohtani T, Sakata Y, Mano T, Takeda Y, Tamaki S, Tsukamoto Y, Kamimura D, Aizawa Y, Miwa T, Komuro I, Soga T, Yamamoto K. L-Carnitine prevents the development of ventricular fibrosis and heart failure with preserved ejection fraction in hypertensive heart disease. J Hypertens. 2012 Sep;30(9):1834-1844.

6. Yamada K, Kinugasa Y, Sota T, Miyagi M, Sugihara S, Kato M, Yamamoto K. Inspiratory Muscle Weakness is Associated with Exercise Intolerance in Patients with Heart Failure with Preserved Ejection Fraction: a Preliminary Study. J Card Fail. 2015 Oct 23.

7. Yamamoto K. «The Third Man» in Heart Failure--Heart Failure With Reduce Ejection Fraction Evolved From Heart Failure With Preserved Ejection Fraction. Circ J. 2015;79(10):2108-2109.

8. Lang RM, Badano LP, Mor-Avi V, Afilalo J, Armstrong A, Ernande L, Flachskampf FA, Foster E, Goldstein SA, Kuznetsova T, Lancellotti P, Muraru D, Picard MH, Rietzschel ER, Rudski L, Spencer KT, Tsang W, Voigt JU. Recommendations for cardiac chamber quantification by echocardiography in adults: an update from the American Society of Echocardiography and the European Association of Cardiovascular Imaging. J Am Soc Echocardiogr. 2015 Jan;28(1):1-39 e14.

9. Chinda J, Nakagawa N, Kabara M, Matsuki M, Endo H, Saito T, Sawada J, Katayama T, Sato N, Hasebe N. Impact of decreased estimated glomerular filtration rate on Japanese acute stroke and its subtype. Intern Med. 2012;51(13):1661-1666.
10. Carter AL, Abney TO, Lapp DF. Biosynthesis and metabolism of carnitine. J Child Neurol. 1995 Nov;10 Suppl 2:S3-7.

11. Liu M, Fang F, Yu CM. Noncardiac comorbidities in heart failure with preserved ejection fraction - commonly ignored fact. Circ J. 2015;79(5):954959.

12. Granger CV, Dewis LS, Peters NC, Sherwood CC, Barrett JE. Stroke rehabilitation: analysis of repeated Barthel index measures. Arch Phys Med Rehabil. 1979 Jan;60(1):14-17.

13. Martin-Sanchez FJ, Gil V, Llorens P, Herrero P, Jacob J, Fernandez C, Miro $\mathrm{O}$, Acute Heart Failure Working Group of the Spanish Society of Emergency Medicine Investigation G. Barthel Index-Enhanced Feedback for Effective Cardiac Treatment (BI-EFFECT) Study: contribution of the Barthel Index to the Heart Failure Risk Scoring System model in elderly adults with acute heart failure in the emergency department. J Am Geriatr Soc. 2012 Mar;60(3):493-498.

14. Nagueh SF, Appleton CP, Gillebert TC, Marino PN, Oh JK, Smiseth OA, Waggoner AD, Flachskampf FA, Pellikka PA, Evangelista A. Recommendations for the evaluation of left ventricular diastolic function by echocardiography. J Am Soc Echocardiogr. 2009 Feb;22(2):107-133.

15. Izawa KP, Watanabe S, Hirano Y, Yamamoto S, Oka K, Suzuki N, Kida K, Suzuki K, Osada N, Omiya K, Brubaker PH, Shimizu H, Akashi YJ. The relation between Geriatric Nutritional Risk Index and muscle mass, muscle strength, and exercise capacity in chronic heart failure patients. Int J Cardiol. 2014 Dec 20;177(3):1140-1141.

16. Crentsil V. Mechanistic contribution of carnitine deficiency to geriatric frailty. Ageing Res Rev. 2010 Jul;9(3):265-268.

17. Costell M, O'Connor JE, Grisolia S. Age-dependent decrease of carnitine content in muscle of mice and humans. Biochem Biophys Res Commun. 1989 Jun 30;161(3):1135-1143.

18. Shah AM, Claggett B, Sweitzer NK, Shah SJ, Anand IS, Liu L, Pitt B, Pfeffer MA, Solomon SD. Prognostic Importance of Impaired Systolic Function in Heart Failure With Preserved Ejection Fraction and the Impact of Spironolactone. Circulation. 2015 Aug 4;132(5):402-414.

19. Study on propionyl-L-carnitine in chronic heart failure. Eur Heart J. 1999 Jan;20(1):70-76

20. Serati AR, Motamedi MR, Emami S, Varedi P, Movahed MR. L-carnitine treatment in patients with mild diastolic heart failure is associated with improvement in diastolic function and symptoms. Cardiology. 2010;116(3):178-182.

21. Koeth RA, Wang Z, Levison BS, Buffa JA, Org E, Sheehy BT, Britt EB, Fu X, Wu Y, Li L, Smith JD, DiDonato JA, Chen J, Li H, Wu GD, Lewis JD, Warrier M, Brown JM, Krauss RM, Tang WH, Bushman FD, Lusis AJ, Hazen SL. Intestinal microbiota metabolism of L-carnitine, a nutrient in red meat, promotes atherosclerosis. Nat Med. 2013 May;19(5):576-585.

22. Troseid M, Ueland T, Hov JR, Svardal A, Gregersen I, Dahl CP, Aakhus S, Gude E, Bjorndal B, Halvorsen B, Karlsen TH, Aukrust P, Gullestad L, Berge RK, Yndestad A. Microbiota-dependent metabolite trimethylamine-N-oxide is associated with disease severity and survival of patients with chronic heart failure. J Intern Med. 2015 Jun;277(6):717-726.

23. Takahashi M1, Ueda S, Misaki H, Sugiyama N, Matsumoto K, Matsuo N, Murao S. Carnitine determination by an enzymatic cycling method with carnitine dehydrogenase. Clin Chem. 1994 May;40(5):817-21. 